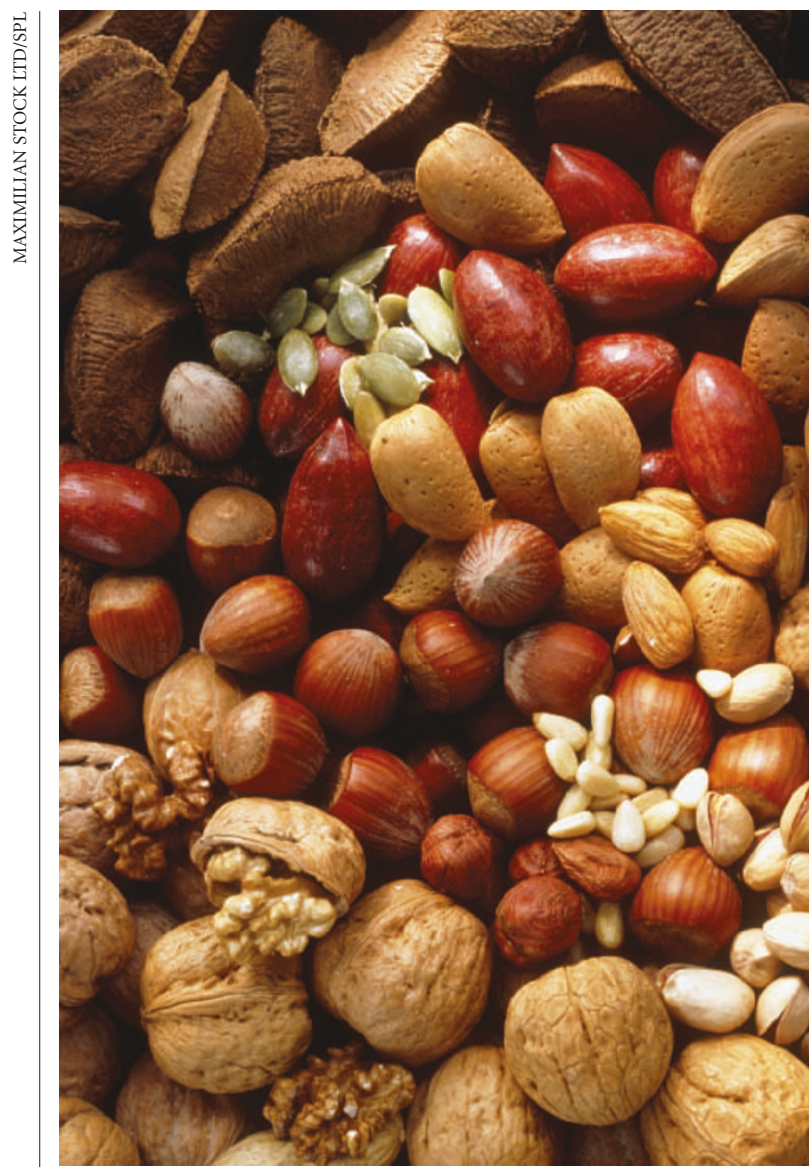

Figure 1 Nuts: why do brazils always rise to the top?

manuscript editor at Physical Review Letters conscientiously (if sceptically) tested the effect in his office using a jar of sand, a large plastic pin (a light intruder) and a steel nut (a heavy intruder). Since this impromptu confirmation, particle-dynamics simulations (subsequently validated experimentally ${ }^{8}$ ) have verified that the reverse brazil nut $(\mathrm{RBN})$ effect appears under ideal in silico conditions, and that multiple intruders also separate in the curious RBN manner ${ }^{9}$.

In fact the RBN effect turned out to be even more complex than realized at first. Subsequent experiments showed that there are actually separate size and density influences at work in a tapped bed ${ }^{10}$. On the one hand, for intruders of a fixed density there is a distinct size threshold above which intruders rise, and below which they sink. On the other hand, intruders of a fixed size rise with a speed that grows and then diminishes non-monotonically as the intruder density is increased. To complicate matters still further, there have been numerous commentaries on the RBN effect, some of which question whether the effect even exists, or whether it is actually a computational $\operatorname{artefact}^{11}$.

Huerta and Ruiz-Suárez ${ }^{3}$ have shown that there are actually two distinct regimes of segregation: one seen at higher frequencies of vibration $(50 \mathrm{~Hz})$, in which the bed becomes fluidized and ordinary buoyancy prevails (heavy intruders sink but light ones float); the other at low frequencies $(5 \mathrm{~Hz})$, in which intruder inertia and bed convection conspire to produce either the ordinary or the reverse brazil nut effect, depending on intruder size and density. This result concurs with much earlier findings that there is a transition at vibration frequencies of about $20 \mathrm{~Hz}$, below which dissimilar-size particles segregate and above which they mix $^{12}$.

Interestingly, this transition coincides with the frequency at which the surface first forms heaps driven by air flow (pumped by pistonlike container motion against the granular bed), suggesting that the transition between ordinary buoyancy and the RBN effect is tied to air flow. This hypothesis has also been investigated recently, but the dual roles of grain and air dynamics remain entangled $^{10,13}$. Huerta and RuizSuárez ${ }^{3}$ propose that this entanglement might only occur in beds of very small particles, which seems to agree with the in silico air-free duplications of the effect. But this leaves us with enduring difficulties in understanding the interplay between intruder size and density — and now bed particle size and vibration speed — and why, to begin with, convection does not prevail to entrain intruders irrespective of such details.

The RBN effect is sure to provide fruit for future exploration and debate. We find ourselves facing the situation anticipated by Mark Twain: "The researches of many commentators have already thrown much darkness on this subject, and it is probable that, if they continue, we shall soon know nothing at all about it." Although farmers can count on continuing harvests of heavy boulders under any segregation model, it remains to be seen whether - and how - pharmaceutical engineers should expect their granular formulations to mix or separate.

Troy Shinbrot is in the Department of Biomedical

Engineering, Rutgers University, Piscataway,

New Jersey 08854, USA.

e-mail: shinbrot@soemail.rutgers.edu

1. Walker, J. The Flying Circus of Physics 72 (Wiley, New York, 1975).

2. Muzzio, F. J., Glasser, B. J. \& Shinbrot, T. Powder Technol. 124, 1-7 (2002).

3. Huerta, D. A. \& Ruiz-Suárez, J. C. Phys. Rev. Lett. 92, 114301 (2004).

4. Knight, J. B., Jaeger, H. M. \& Nagel, S. R. Phys. Rev. Lett. 70, 3728-3731 (1993).

5. Ehrichs, E. E. et al. Science 267, 1632-1634 (1995).

6. Gallas, J. A. C., Herrmann, H. J., Pöschel, T. \& Sokolowski, S. J. Stat. Phys. 82, 443-450 (1996)

7. Shinbrot, T. \& Muzzio, F. J. Phys. Rev. Lett. 81, 4365-4368 (1998)

8. Breu, A. P. J., Ensner, H.-M., Kruelle, C. A. \& Rehberg, I. Phys. Rev. Lett. 90, 014302 (2003).

9. Hong, D. C., Quinn, P. V. \& Luding, S. Phys. Rev. Lett. 86, 3423-3426 (2001).

10. Möbius, M. E., Lauderdale, B. E., Nagel, S. R. \& Jaeger, H. M. Nature 414, 270 (2001)

11. Canul-Chay, G. A., Belmont, P. A., Nahmad-Molinari, Y. \& Ruiz-Suárez, J. C. Phys. Rev. Lett. 89, 189601 (2002).

12. Brone, D. \& Muzzio, F. J. Phys. Rev. E 56, 1059-1063 (1997).

13. Yan, X., Shi, Q., Hou, M. \& Lu, K. Phys. Rev. Lett. 91, 014302 (2003).

\title{
Genome sequencing
}

\section{Differences with the relatives}

\author{
Jean Weissenbach
}

\section{One of the chimpanzee's chromosomes has been sequenced to} near-completion. What can this accomplishment tell us about how we have come to look and act so differently from our chimp relatives?

$\mathrm{T}$ here are good reasons to continue the endeavour to accumulate genome sequence data from the passengers of Noah's Ark. As illustrated on page 382 of this issue ${ }^{1}$, genome sequences can serve to address basic evolutionary issues — the power of this approach depending to a large extent on the amount and quality of data available.

The rationale for sequencing the genome of the chimpanzee (Pan troglodytes; Fig. 1, overleaf) has been explained on numerous occasions (see ref. 2 for a review), and a publicly funded effort, involving some of the large US sequencing centres, has already produced a draft assembly of the whole sequence $^{3}$. But this initial assembly still contains many gaps and ambiguities that present difficulties for some types of analysis.

In an independent effort ${ }^{1}$, a consortium of Old World humans has now sequenced chimpanzee chromosome 22 to a degree of completion and accuracy equivalent to that of the human genome assembly in its present version. The quality of this chimp chromosome sequence is therefore good enough to allow reliable comparisons with its human counterpart (chromosome 21). A chimpanzee chromosome provides a unique angle from which to look at the human genome and to draw conclusions about its recent evolution, because the sequences of 
these evolutionary near-neighbours started drifting apart some six million years ago. The longer-term hope, of course, is to identify those sequence changes that could account for the present-day physical, physiological and behavioural differences between chimps and people.

By lining up chimp chromosome 22 and human chromosome 21 and comparing them nucleotide by nucleotide, the consortium found instances in which one nucleotide was substituted for another in only about $1.44 \%$ of the sequence. The chimpanzee chromosome has been sequenced to an accuracy of less than one error in $10^{4}$ bases, so sequencing mistakes account for less than $1 \%$ of the observed single-nucleotide mismatches. There is also an impressive number $(68,000)$ of small to large stretches of DNA that have been either gained or lost (these are called 'insertions or deletions', 'indels' for short) in one species or the other.

The number of single-nucleotide substitutions is in the range found in earlier studies, but the frequency and size of the indels are more of a surprise. Although most of the indels are less than 30 nucleotides long, some attain sizes of up to 54,000 nucleotides. Those of about 300 nucleotides or more frequently involve transposable elements - DNA sequences that multiply and insert new copies of themselves throughout a genome. For a subset of these 300-nucleotide-plus indels, the authors were able to extend the comparison to other great apes: gorillas and orang-utans. They could thereby infer the lineage (chimp or human) in which the alterations occurred, and could distinguish between insertions and deletions that is, whether a given sequence was added in one lineage or deleted in the other. These comparisons show that insertions of about 300 nucleotides, mainly of the type of transposable element known as an Alu repeat, have occurred preferentially in the human lineage. Deletions and other insertions seem to have occurred at similar frequencies in both lineages.

One of the strongest arguments in support of the chimpanzee genome project was always that having the chimp sequence makes it possible to determine which variants of single nucleotide polymorphisms (SNPs) — single nucleotide differences between individual humans - represent the 'original' form ${ }^{4}$. This information is important in, for instance, genetic association studies aimed at mapping the locations of gene variants associated with complex diseases such as diabetes or high blood pressure. On the basis of the chimp chromosome 22 sequence, the consortium determined the

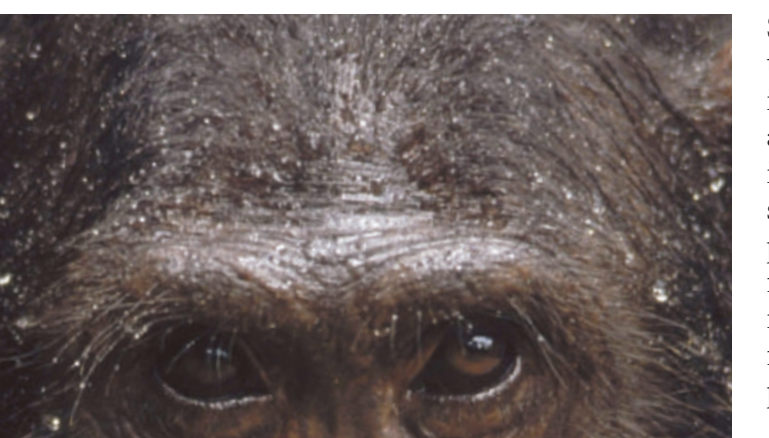

Surprisingly, however, the consortium found that sequence differences in the protein-coding regions of genes are not a great deal less common than in non-coding genomic regions. But some of the affected genes might be pseudogenes - defective copies of functional genes - that have arisen recently. And, among 231 presumably functional genes that could be compared between chimps and humans, 179 have protein-coding regions of identical length; 140 of the predicted encoded proteins would differ by one amino acid or more, but probably with little or no functional impact. Of the other 52 genes, however, 47 show more significant structural changes.

The consortium could not resist making preliminary studies of the expression of the genes on human chromosome 21 and chimp chromosome 22 as well. Their analyses indicate that - looking at just two tissues — about $20 \%$ of these genes show significant variations in their expression. Extrapolation from these findings suggests that if this chromosome represents about $1 \%$ of mammalian genes, there may well be thousands of genes that either encode an altered protein or are expressed differentially in humans and chimpanzees. This

ancestral form of some 20,000 SNPs from human chromosome 21 (although, given that most of the chromosome 22 sequence has come from just one chimpanzee, it remains formally possible that some of the same polymorphisms also occur in chimp populations). The comparison shows that, as expected, transitions (mutation of one purine nucleotide, adenine or guanine, to the other, or of one pyrimidine nucleotide, cytosine or thymine, to the other) are more frequent SNPs than are transversions (mutations of a purine to a pyrimidine and vice versa). Moreover, mutation occurs at guanines and cytosines more frequently than at adenines and thymines.

In searching for the basis of the physical variation between chimps and humans, differences in genome sequences are just the first place to start: we then need to know what these differences mean. Many of the sequence variations might have no effect at all. Those that do might occur in nonprotein-coding parts of the genome or in control genes, thereby influencing the level, location and timing of gene expression. Other changes might alter the sequences of encoded proteins, resulting in loss or gain of function. And entire genes might be deleted or acquired in one lineage or another.

Given the broad similarities between chimps and humans, many researchers thought that changes that alter aminoacid sequences would not be very frequent. will not simplify the search for the hypothetical key genetic changes that prevented us from remaining as apes.

Even if the major physical, physiological and behavioural differences between the two species do not result simply from an accumulation of many small alterations, the challenge to find the most crucial changes is still ahead. For example, the FOXP2 gene product, which is important for language development, differs by two amino acids in humans and chimps, suggesting that the gene has been a target of selection in the human lineage. Yet the role of this gene in language was suggested not by human-chimp comparisons ${ }^{5}$ but by mutation studies in humans ${ }^{6}$.

Identifying sequence changes in the chimpanzee lineage that are likely to have been irrelevant to the acquisition of humanspecific traits will depend on sequence comparisons with other great apes. Do we now need the gorilla genome sequence to shed more light on the questions raised by comparing human and chimp DNA?

Jean Weissenbach is at Genoscope, 91000 Evry, France.

e-mail: jsbach@genoscope.cns.fr

\footnotetext{
1. The International Chimpanzee Chromosome 22 Consortium Nature 429, 382-388 (2004).

2. Olson, M. \& Varki, A. Nature Rev. Genet. 4, 20-28 (2003).

3. www.genome.gov/11509418

4. Hacia, J. G. et al. Nature Genet. 22, 168-171 (1999).

5. Enard, W. et al. Nature 418, 869-872 (2002).

6. Lai, C. S. L., Fisher, S. E., Hurst, J. A., Vargha-Khadem, F. \& Monaco, A. P. Nature 413, 519-523 (2001).
} 